\title{
Conversion of Adjustable Gastric Banding to Roux-en-Y Gastric Bypass in One or Two Steps: What Is the Best Approach? Analysis of a Multicenter Database Concerning 832 Patients
}

\author{
J. Pujol-Rafols ${ }^{1}$ • A. I. Al Abbas ${ }^{2}$ - S. Devriendt ${ }^{3}$ • A. Guerra ${ }^{4}$ - M. F. Herrera ${ }^{5}$ - J. Himpens ${ }^{3} \cdot$ E. Pardina $^{6} \cdot$ S. Pouwels $^{7} \cdot$ \\ A. $\operatorname{Ramos}^{8} \cdot$ R. J. Ribeiro ${ }^{9} \cdot$ B. Safadi ${ }^{2} \cdot$ H. Sanchez-Aguilar ${ }^{5} \cdot$ C. de Vries $^{10} \cdot$ B. Van Wagensveld ${ }^{11}$
}

Received: 3 May 2020 / Revised: 21 August 2020 / Accepted: 26 August 2020 / Published online: 3 September 2020

(C) Springer Science+Business Media, LLC, part of Springer Nature 2020

\begin{abstract}
Background Roux-en-Y gastric bypass (RYGB) is often the preferred conversion procedure for laparoscopic adjustable gastric banding (LAGB) poor responders. However, there is controversy whether it is better to convert in one or two stages. This study aims to compare the outcomes of one and two-stage conversions of LAGB to RYGB.

Methods Retrospective review of a multicenter prospectively collected database. Data on conversion in one and two stages was compared.

Results Eight hundred thirty-two patients underwent LAGB conversion to RYGB in seven specialized bariatric centers. Six hundred seventy-three (81\%) were converted in one-stage. Patients in the two-stage group were more likely to have experienced technical complications, such as slippage or erosions $(86 \%$ vs. $37 \%, p=0.0001)$ and to have had a higher body mass index $(\mathrm{BMI})$ $\left(41.6\right.$ vs. $\left.39.9 \mathrm{Kg} / \mathrm{m}^{2}, p=0.005\right)$. There were no differences in postoperative complications and mortality rates between the onestage and two-stage groups ( $13.5 \%$ vs. $10.8 \%$, and $0.7 \%$ vs. $0.0 \%$ respectively, $p=n s)$. Mean final BMI and \%total weight loss (\%TWL) for the one-stage and the two-stage groups were $31.6 \mathrm{vs} .32 .4 \mathrm{Kg} / \mathrm{m}^{2}(p=\mathrm{ns})$ and $30.4 \mathrm{vs} .26 .8(p=0.017)$ after a mean follow-up of 33 months. Follow-up at 1, 3, and 5 years was $98 \%, 75 \%$, and $54 \%$, respectively.

Conclusions One-stage conversion of LAGB to RYGB is safe and effective. Two-stage conversion carries low morbidity and mortality in the case of band slippage, erosion, or higher BMI patients. These findings suggest the importance of patient selection when choosing the appropriate conversion approach.
\end{abstract}

Keywords LAGB · Gastric banding · Gastric bypass · RYGB · Revision · Revisional surgery · Conversion

\section{Introduction}

Laparoscopic adjustable gastric banding (LAGB), firstly performed by Cadière in 1992, became widely performed during

J. Pujol-Rafols

juan.pujol.rafols@gmail.com

1 Clínica Mi Tres Torres, Barcelona, Spain

2 American University of Beirut Medical Center (AUBMC), Beirut, Lebanon

3 AZ Sint Blasius, Dendermonde, Belgium and CHIREC Hospitals Brussels, Auderghem, Belgium

4 Centro Hospitalar de Lisboa Central, Lisbon, Portugal

5 Instituto Nacional de Nutrición, Centro Médico ABC, México City, México the 1990s. Its technical simplicity, reversibility, and low acute complication rate were major factors in its popularity. Despite losing prominence over the past two decades, LAGB remains the third most performed technique worldwide, only

6 Departament de Bioquímica i Biomedicina Molecular, Facultat de Biologia, Universitat de Barcelona, Barcelona, Spain

7 Department of Intensive Care Medicine, Elisabeth-Tweesteden Hospital, Tilburg, The Netherlands

8 Gastro-Obeso-Center - Advanced Institute In Bariatric And Metabolic Surgery, Sao Paulo, Brazil

9 Departamento de Cirugia Geral e do Centro Multidisciplinar da Doença Metabólica, CLISA-Lusiadas, Amadora, Portugal

10 OLVG-West, Amsterdam, the Netherlands

11 Quro Obesity Center, Dubai, United Arab Emirates 
surpassed by Roux-en-Y gastric bypass (RYGB) and sleeve gastrectomy [1]. As a result, there are currently thousands of patients with bands implanted around the world.

While some authors defend the benefits of LAGB [2], many others have highlighted the relatively high rate of poor responders and technical complications discovered during medium and long-term follow-up [3, 4]. A significant number of patients with LAGB do not achieve satisfactory weight loss, and some other patients experience technical problems related to the device. Most of them undergo further surgery to remove the band, and in many cases, patients are offered an alternative surgical option in order to prevent weight regain. Conversion from LAGB to RYGB is the mainstay for many surgeons because of its low complication rate and good weight loss results $[5,6]$. Although there is a great number of published studies about LAGB conversion to RYGB, there is still controversy whether to perform the procedure in onestage (remove the band and perform the conversion to RYGB at the same time) or in two stages (remove the band and perform RYGB at a later date). No consensus exists among the scientific community on this issue. While the American Society for Metabolic and Bariatric Surgery (ASMBS) revision surgery task force published a systematic review in 2014 indicating that two-step revision of LAGB to RYGB is safer than a one-step approach [7], there are multiple reports in the literature citing that a single-step RYGB conversion is safe except when there is intra-gastric band erosion [6, 8-11].

The aim of this study is to compare the short-term complications and mortality rates between one- and two-staged conversions of LAGB to RYGB. The secondary aim is to compare weight loss outcomes at the last available follow-up between the two approaches.

\section{Material and Methods}

Seven different centers around the world, with extensive experience in bariatric surgery, were asked to collate data from their individual prospectively collected registries. They provided data on all patients who had been converted owing to LAGB unsatisfactory results. The pooled data were standardized and merged in an electronic database. We have previously published the first report using this database to compare outcomes of different revisional procedures [12]. For the current study, we identified the patients who underwent conversion of LAGB to RYGB and grouped them into one-staged vs. two-staged approaches.

\section{Surgical Technique}

All revisional LAGB-to-RYGB procedures analyzed in this study were performed following standard technical recommendations and have been described in our earlier publication
[12]. In summary, if the band was still in place, the procedure began by opening the entire anterior gastro-gastric plication that covered the band before its removal. To perform the RYGB, the volume of the gastric pouch was tailored between 20 and $50 \mathrm{~mL}$. The gastrojejunal anastomosis was either constructed using a linear stapler or was hand-sewn depending on the surgeon's preference. A side-to-side linear stapled jejunojejunostomy was constructed, leaving an ante-colic and ante-gastric alimentary limb, measuring between 60 and $150 \mathrm{~cm}$ and a biliopancreatic limb of about 50 to $70 \mathrm{~cm}$.

The decision to perform the procedure in one versus two stages was most frequently taken preoperatively, based on individual and institutional preferences in the face of the actual indication for conversion. In rare occasions, such as unexpected local findings or technical difficulties, the scheduled one-stage strategy was abandoned.

\section{Outcome Measures}

For the present study, the following data was assessed:

1- Subjects' demographic and medical data: including age, gender, height, preoperative weight (before LAGB), date of LAGB, date of LAGB removal, date when the revisional procedure was performed, weight immediately before conversion to RYGB, and last weight as measured during patient's follow-up.

2- The main reason for conversion (to choose between two different options): unsatisfactory weight loss or devicerelated technical problems (such as band slippage or erosion). In the case of a patient experiencing both a technical problem and unsatisfactory weight loss, the surgeon who collected the data was requested to select the clinically most relevant.

3- Major complications: defined as any complication that resulted in a prolonged hospital stay ( $>7$ days), administration of an anticoagulant, reoperation, or re-intervention (based on the recommendations of the American Society for Metabolic and Bariatric Surgery Clinical Issues Committee) [13]. Special emphasis was made to identify leaks (defined as an effluent of gastrointestinal content through a suture line that may collect near the anastomosis or exit through the wall or the drain), hemorrhage (considered clinically relevant when patients required reintervention or transfusion), and operative mortality (defined as any death, regardless of cause, occurring within 30 days after surgery in or out of the hospital).

Patients were considered lost to follow-up if they failed to attend 2 consecutive medical appointments.

Weight was measured in kilograms and height in meters. Initial body mass index ( $\left.{ }_{\mathrm{i}} \mathrm{BMI}\right)$, final body mass index ( $\left.{ }_{\mathrm{f}} \mathrm{BMI}\right)$, change in BMI $(\Delta \mathrm{BMI})$, percentage of excess BMI loss 
(\%EBMIL), and percentage of total weight loss (\%TWL) were calculated based on the following formulas:

$\cdot{ }_{\mathrm{i}} \mathrm{BMI}=$ initial weight $/$ height $^{2}$

- ${ }_{\mathrm{f}} \mathrm{BMI}=$ last weight $\mathrm{recorded} /$ height $^{2}$

- $\Delta \mathrm{BMI}=\left({ }_{\mathrm{i}} \mathrm{BMI}\right)-\left({ }_{\mathrm{f}} \mathrm{BMI}\right)$

•\% EBMIL $=\left[\Delta \mathrm{BMI} /\left({ }_{\mathrm{i}} \mathrm{BMI}-25\right)\right] \times 100$

•\% TWL $=[($ initial weight-final weight $) /$ initial weight $] * 100$

Satisfactory weight loss was defined as \%EBMIL > 50 according to Reinhold weight loss classification [14].

\section{Statistical Analysis}

Data are expressed as mean \pm standard deviation for continuous variables and as the total number of events and percentages for qualitative ones. Unpaired $t$ test was used to compare continuous variables and chi-squared test was used for the categorical variables. $P<0.05$ was considered significant in both cases. Statistical analysis was performed using GraphPad Prism (version 8, San Diego, CA, USA).

\section{Results}

A total of 832 patients underwent revision from LAGB to RYGB from March 2002 to January 2018. Six hundred and seventy-three $(80.9 \%)$ of the cases were one-stage procedures, while $159(19.1 \%)$ were two-stage procedures. In the case of two-stage conversions, the mean time lapse between the removal of the band and the performance of the RYGB was $34.4 \pm 35.9$ months.

Follow-up rates at 1, 3, and 5 years were 98\%, 75\%, and $54 \%$, respectively. For those patients who were lost to followup before their final time-point, the last weight recorded was considered final weight.

Mean age of the whole group at the time of conversion was $43.5 \pm 10.2$ years, and $696(84 \%)$ participants were female. The mean initial weight (when LAGB was performed) was $123.2 \pm$ $22.6 \mathrm{Kg}$ representing a mean initial BMI of $44.3 \pm 6.9 \mathrm{Kg} / \mathrm{m}^{2}$. Overall, mean weight and BMI at the time of conversion to RYGB were $112.1 \pm 22.2 \mathrm{Kg}$ and $40.2 \pm 6.9 \mathrm{Kg} / \mathrm{m}^{2}$. There were no statistical differences regarding gender, age, initial weight, and initial BMI between the patients who were converted in onestage and those who were converted in two stages. However, patients in the two-stage group tended to weigh more and had significantly higher BMI at the time of conversion (Table 1).

\section{Reasons for Conversion}

A definitive reason for conversion was provided for 815 patients $(97.9 \%)$. Overall, 442 patients $(54.2 \%)$ had to be re-
Table 1 Demographic data

\begin{tabular}{llll}
\hline & One-stage & Two stages & $p$ \\
\hline$N$ & 673 & 159 & \\
Age at LAGB & $37.3 \pm 10.2$ & $36.8 \pm 9.9$ & 0.5378 \\
Age at RYGB & $43.4 \pm 10.3$ & $43.9 \pm 9.7$ & 0.5792 \\
Gender (male/female) & $16 \% / 84 \%$ & $17 \% / 83 \%$ & 0.8098 \\
Initial weight at LAGB & $123.6 \pm 22.9$ & $121.8 \pm 21.2$ & 0.3781 \\
Initial BMI at LAGB & $44.3 \pm 7.0$ & $44.0 \pm 6.4$ & 0.6301 \\
Weight at RYGB & $111.4 \pm 22.4$ & $115.0 \pm 21.4$ & 0.0665 \\
BMI at RYGB & $39.9 \pm 6.9$ & $41.6 \pm 6.4$ & 0.0048 \\
\hline
\end{tabular}

$B M I$ body mass index

$L A G B$ laparoscopic adjustable gastric band

$R Y G B$ Roux-en-Y gastric bypass

operated on because of unsatisfactory weight loss or weight regain. Conversely, 373 patients $(45.8 \%)$ had to be reoperated on owing to technical complications associated with the band (such as band slippage or intragastric band migration).

Most of the patients in the one-stage group were converted owing to unsatisfactory weight loss or weight regain (421, $63.5 \%$ ). By contrast, most of the patients in the two-stage group were converted owing to band-related technical problems $(131,86.2 \%)$. The proportion "patients who had to be converted for unsatisfactory weight loss versus patients who had to be converted due to technical problems" differed significantly between the one-stage and two-stage groups, ( $p=$ 0.0001) (Table 2).

\section{Complications and Mortality}

A total of 108 patients (13.0\%) suffered postoperative complications. Overall, complication rates, leak rates, and bleeding episodes were not significantly different between the group of patients operated in one-stage and those operated in two stages. Five patients died, 4 in-hospital (1 due to leak, 1 due to bleeding, 1 due to cardiac tamponade, 1 due to pulmonary thromboembolism) and 1 outside hospital (due to an unknown cause). All fatalities were in the one-stage group, whereas no patient died in the two-stage group (Table 3 ). The mortality rate $(0.7 \%)$ in the one-stage group was not statistically different from that in the two-stage group.

\section{Weight Loss Outcomes}

Overall, mean weight dropped from $112.1 \mathrm{Kg}$ at the time of conversion to $88.6 \mathrm{Kg}$ after a mean follow-up of $33.1 \pm$ 31.4 months. Mean final BMI was $31.8 \mathrm{Kg} / \mathrm{m}^{2}$ for the entire group. Mean final \%EBMIL and mean final \%TWL were significantly higher in the group of patients converted in 
Table 2 Reasons for conversion

\begin{tabular}{lllll}
\hline & One-stage & Two stages & $p$ & Total \\
\hline IWL/tech. problems & $63.5 \% / 36.5 \%$ & $13.8 \% / 86.2 \%$ & 0.0001 & $54.2 \% / 45.8 \%$ \\
\hline
\end{tabular}

IWL insufficient weight loss, Tech technical

one-stage (Table 4). Four hundred and fifty-seven patients (75.4\%) in the one-stage group and one hundred and three patients $(68.2 \%)$ in the two-stage group achieved \%EBMIL $>50$; this difference was not statistically significant $(p=$ 0.0712).

\section{Discussion}

The rate of gastric band removal increases with time after the surgery with percentages ranging from 7.5 to $71 \%$ [2-4, 15-21]. For many surgeons, RYGB is the preferred followon procedure after unsuccessful LAGB [5, 6]. However, there is still controversy about whether the full procedure (LAGB removal and conversion to RYGB) should be done in one time or in two separate phases.

Performing the conversion in one-stage has certain advantages: the patient needs to be hospitalized only once; it requires a single anesthetic intervention and a single operation. Importantly, it avoids waiting time and prevents possible weight regain during the time-gap between the first and the second operation. Lanthaler et al., for example, reported an increase in the patients' BMI from an initial $29.6 \mathrm{Kg} / \mathrm{m}^{2}$ at band removal to a $38.2 \mathrm{Kg} / \mathrm{m}^{2}$ before the conversion operation, after a mean waiting time of 1.2 years [22].

We extracted and analyzed data from a multi-center database of 832 consecutive patients who underwent conversion of LAGB to RYGB to determine if there were any differences in short- and long-term outcomes between one-stage and two-stage approaches. We also aimed to compare possible differences in the two groups to detect any potential selection criteria that led the surgeons to choose one approach versus the other.

Our data suggests that surgeons prefer to choose the onestage conversion whenever possible, as $81 \%$ of the patients were treated this way. Revisional surgery in one-stage was mainly performed in patients who did not achieve satisfactory

Table 3 30-day postoperative complications

\begin{tabular}{lllll}
\hline & One-stage & Two stages & $p$ & Total \\
\hline Compl. rate & $91 / 67313.5 \%$ & $17 / 15910.8 \%$ & 0.3397 & $108 / 83213.0 \%$ \\
Leaks & $25 / 6733.7 \%$ & $7 / 1594.5 \%$ & 0.6850 & $32 / 8323.8 \%$ \\
Bleeding & $22 / 6733.3 \%$ & $5 / 1593.2 \%$ & 0.9366 & $27 / 8323.2 \%$ \\
Mortality & $5 / 6730.7 \%$ & $0 / 1590.0 \%$ & 0.2746 & $5 / 8320.6 \%$ \\
\hline
\end{tabular}

Compl. complication weight loss with the band. The majority (63.5\%) of the onestage procedures were performed for weight issues and only $36.5 \%$ were performed due to technical problems with the banding.

By contrast, the two-stage approach seemed to be a very popular option of conversion in more complex patients, who had to be re-operated on owing to technical problems with the band. Most (86.2\%) of the two-stage approach group underwent conversion to RYGB due to technical problems, such as band slippage, gastric pouch dilation, intra-gastric band migration, food intolerance, or esophageal motility problems. Only $13.8 \%$ of the patients converted in two stages were operated for unsatisfactory weight loss solely. In addition, the patients in this latter group weighed more at the time of conversion compared with the one-stage approach patients. These findings are in line with Theunissen et al.'s experience, whose one-stage redo procedures were mainly indicated in the case of weight regain and vomiting or intolerance of the LAGB, whereas the two-stage procedures were most frequently performed in the case of device-related complications (slippage, erosion) [8]. In fact, in the case of band erosion, the vast majority of surgeons appear to opt for a two-stage conversion $[6,9,23]$.

In our analysis, the rates of major complications, leakage, and bleeding episodes after the conversion procedure were similar in the two groups. There were five mortalities $(0.7 \%)$ in the one-stage approach and none in the two-stage, for a total cumulative mortality of $0.6 \%$. While this difference does not reach statistical significance, it is nonetheless a disturbing trend that should be taken into account. Revisional surgery is always a challenging operation. The surgeon who decides to convert in one-stage should be cautious and not hesitate to switch to a two-stage approach if the case warrants it.

The complication rate in this study is in line with what has been published in re-operative bariatric surgical literature [6-9, 24-30] (Tables 5 and 6).

Table 4 Weight loss outcomes

\begin{tabular}{lllll}
\hline & One-stage & Two stages & $p$ & Total \\
\hline Final weight & $88.3 \pm 19.5$ & $89.7 \pm 18.3$ & 0.4132 & $88.6 \pm 19.2$ \\
\%EBMIL & $67.7 \pm 31.7$ & $62.1 \pm 28.9$ & 0.0486 & $66.6 \pm 31.2$ \\
\%TWL & $30.4+/ 17.5$ & $26.8+/ 13.2$ & 0.0177 & $29.7+/ 16.8$ \\
fBMI & $31.6+/-6.2$ & $32.4 \pm 5.7$ & 0.1828 & $31.8 \pm 6.1$ \\
\hline
\end{tabular}

$\% E B M I L$ percent excess body mass index loss, $\% T W L$ percent total weight loss, $\beta B M I$ final body mass index (in $\mathrm{Kg} / \mathrm{m}^{2}$ ) 
Table 5 Selected papers reporting LAGB to RYGB conversion in one-stage

\begin{tabular}{lrllll}
\hline Author & $N$ & Compl. (\%) & Leak (\%) & Bleeding (\%) & Death (\%) \\
\hline Van Nieuwenhove $^{24}$ & 23 & 22.0 & 0.0 & 4.3 & 0.0 \\
Robert $^{25}$ & 71 & & 0.0 & & \\
Fournier $^{6}$ & 387 & & 1.8 & 0.5 & \\
Theunissen $^{8}$ & 71 & 16.9 & & & 0.0 \\
Ardestani $^{26}$ & 19 & 10.5 & & 0.0 & 0.0 \\
Carr $^{27}$ & 51 & 1.96 & 2.0 & 1.0 & 0.0 \\
Aarts $^{9}$ & 195 & 4.0 & 1.0 & 0.0 & 0.7 \\
Chansaenroj $^{28}$ & 9 & 11.1 & 0.0 & 3.3 & 0.5 \\
Present series $_{\text {TOTAL }}$ & 673 & 13.5 & 3.7 & 2.0 & \\
\hline
\end{tabular}

$N$ number of patients, Compl. complications, $N R$ not reported
Multiple papers support our findings; Dang et al. systematically reviewed the literature comparing the safety of onestep versus two-step revisional bariatric surgery from LAGB to RYGB or sleeve gastrectomy. Eleven studies and 1370 patients were included. Meta-analysis found comparable rates of complications, morbidity, and mortality between the onestage and two-stage procedures for both RYGB and SG groups. They concluded that immediate or delayed revisional bariatric surgeries are both safe options for LAGB revision [11]. Theunissen et al. also published their results on band conversion to RYGB in 107 patients, and found a very similar complication rate among those patients operated on in one or two stages ( 16.9 vs. $16.7 \%$; $p=n s$ ). They concluded by recommending performing the conversion in one-stage except in the case of an acute complication of the LAGB [8]. Fournier et al., analyzing their extensive experience with 642 patients converted from LAGB to RYGB, also found similar complication rates whether they converted in one-stage or in two stages ( 10.2 vs. $9.1 \%$ resp. $p=0.68)$ with similar rates of leaks and bleeding episodes. They also concluded that, in experienced hands, a one-stage approach is safe in most cases, and does not increase the complication rate, but they still recommended performing the operation in two stages in the case of band erosion. [6]. Based on our own findings, we also believe that the best way to reduce morbidity is to decide to proceed in one or in two stages based on the patient's characteristics.

Finally, this study shows that some data assessing weight loss (\%EBMIL and \%TWL) tends to be better in patients operated in a single stage. It is possible that, since most of the patients treated in one-stage present with lower BMI at the time of reoperation and have no other added technical complications, their operation may be technically easier. Conversely, fashioning a small gastric pouch is more difficult when there is proximal gastric and esophageal dilatation, in the case of gastric band slippage, or when there is extensive inflammation, in the case of gastric band erosion. These technical issues may account for the differences seen in the final weight loss outcome favoring the one-stage approach.

The present study has several strengths and also some weaknesses. Despite the fact that the data was collected prospectively by each participating center, the data was analyzed retrospectively. As such, data interpretation is subject to the limitations and the selection bias inherent in retrospective data analysis. Because of lack of randomization, patient selection bias may have played a role in the similarity of the outcomes. In some patients, the surgeon had the option to decide for either one- or two-stage conversion; in others, it was the intraoperative conditions that forced to choose the 2-stage procedure. Unfortunately, the database used for this study does
Table 6 Selected papers reporting banding conversion in two stages

\begin{tabular}{llllll}
\hline Author & $N$ & Compl. $(\%)$ & Leak $(\%)$ & Bleeding $(\%)$ & Death (\%) \\
\hline Van Nieuwenhove $^{24}$ & 14 & 0.0 & 0.0 & 0.0 & \\
Fournier $^{6}$ & 255 & & 1.2 & 0.8 & \\
Theunissen $^{8}$ & 36 & 16.7 & & & 0.0 \\
Carr $^{27}$ & 13 & 0.0 & 0.0 & 0.0 & 0.0 \\
Carandina $^{29}$ & 74 & 13.5 & 5.4 & 2.7 & 0.0 \\
Topart $^{30}$ & 32 & 12.5 & 0.0 & 0.0 & 0.0 \\
Present series & 159 & 10.8 & 4.5 & 3.5 & 0.0 \\
TOTAL & 583 & 11.3 & 2.6 & 1.7 & \\
\hline
\end{tabular}

$N$ number of patients, Compl. complications 
not specify whether the decision to convert to one or two stages was made before or during the operation. It also does not specify which kind of technical problem each particular patient had. It is not easy to merge these data together in a multicenter study. Terminology such as "slippage," "pouch dilation," "band intolerance," "dysphagia," and "band dislocation" may have been defined and classified differently in the different centers taking part of the study.

The patients' follow-up rate decreased with time, especially from the third year onwards. This is an inevitable flaw in any study analyzing long-term data, but while it could have some influence on the weight loss results, it hardly if at all skews the post-operative complications' rate. Finally, this study evaluated the rate of complications and weight loss, but not comorbidity resolution. The multicentricity of the data prevented us to merge data on co-morbidity resolution because there were many methodological differences in reporting across the participating centers. Future-randomized control trials may be necessary for evaluation of this very issue.

However, the main strength of this study is that it pertains to a large sample from seven experienced surgical programs in different countries. This avoids the bias of single center series and dilutes factors that may be related to a single surgeon, including patient selection criteria, choice of anastomosis, or certain technical issues such as biliopancreatic limb length in RYGB.

\section{Conclusions}

One-stage conversion from LAGB to RYGB is safe and effective, which is in line with published data that seem to indicate that this strategy is the most commonly used when revising LAGB. One-stage conversion is particularly applicable for managing non-responders in the absence of major mechanical problems. The choice of a two-stage conversion is prudent in the case of band slippage or erosion. By selecting the one- versus the twostage strategy according to the patient's characteristics, in most cases, good results can be achieved, with reasonable surgical morbidity and good long-term weight loss.

Acknowledgments The authors would like to thank Dr. Ahmed Ahmed and Dr. Yitka Graham for their invaluable help in reviewing this manuscript.

\section{Compliance with Ethical Standards}

All procedures performed in the present study were in accordance with the ethical standards of the institutional research committee and with the 1964 Helsinki Declaration and its later amendments or comparable ethical standards. For this type of study, formal consent is not required.

Conflict of Interest J. Himpens receives consulting fees from Ethicon Endosurgery and from Medtronic. Bart A. van Wagensveld is employed by Obesitas International, a strategic alliance partner of Medtronic. All other authors declare that they have no conflict of interest.

\section{References}

1. Ramos A, Kow L, Brawn W, et al. 5th IFSO Global Registry Report, ISBN 978-1-9160207-3-3 Published by Dendrite Clinical Systems Ltd; Sep 2019.

2. O'Brien PE, Hindle A, Brennan L, et al. Long-term outcomes after bariatric surgery: a systematic review and meta-analysis of weight loss at 10 or more years for all bariatric procedures and a singlecentre review of 20-year outcomes after adjustable gastric banding. Obes Surg. 2019;29:3-14.

3. Himpens J, Cadière GB, Bazi M, et al. Long-term outcomes of laparoscopic adjustable gastric banding. Arch Surg. 2011;146(7): 802-7.

4. Naef M, Mouton W, Naef U, et al. Graft survival and complications after laparoscopic gastric banding for morbid obesity-lessons learned from a 12-year experience. Obes Surg. 2010;20(9):120614.

5. Weber M, Müller MK, Michel JM, et al. Laparoscopic roux-en-Y gastric bypass, but not rebanding, should be proposed as rescue procedure for patients with failed laparoscopic gastric banding. Ann Surg. 2003;238:827-34.

6. Fournier P, Gero D, Dayer-Jankechova A, et al. Laparoscopic Roux-en-Y gastric bypass for failed gastric banding: outcomes in 642 patients. Surg Obes Relat Dis. 2016;12(2):231-9.

7. Brethauer SA, Kothari S, Sudan R, et al. Systematic review on reoperative bariatric surgery American Society for Metabolic and Bariatric Surgery Revision Task Force. Surg Obes Relat Dis. 2014;10:952-72.

8. Theunissen CMJ, Nele Guelinckx N, Maring JK, et al. Redo laparoscopic gastric bypass: one-step or two-step procedure? Obes Surg. 2016;26:2675-82.

9. Aarts E, Koehestanie P, Dogan K, et al. Revisional surgery after failed gastric banding: results of one-stage conversion to RYGB in 195 patients. Surg Obes Relat Dis. 2014;10(6):1077-83.

10. Stroh C, Weiner R, Wolff S, et al. One versus two-step Roux-en-Y gastric bypass after gastric banding. Data analysis of the German Bariatric Surgery Registry. Obes Surg. 2015;25(5):755-62.

11. Dang J, Switzer N, Wu J, et al. Gastric band removal in revisional bariatric surgery, one-step versus two-step: a systematic review and meta-analysis. Obes Surg. 2016;26(4):866-73.

12. Pujol Rafols J, Abbas A, Devriendt S, et al. Roux-en-Y gastric bypass, sleeve gastrectomy, or one anastomosis gastric bypass as rescue therapy after failed adjustable gastric banding: a multicenter comparative study. Surg Obes Relat Dis. 2018;14(11):1659-66.

13. Brethauer S, Kim J, el Chaar M, et al. Standardized outcomes reporting in metabolic and bariatric surgery. Obes Surg. 2015;25(4):587-606.

14. Reinhold RB. Critical analysis of long term weight loss following gastric bypass. Surg Gynecol Obstet. 1982;155(3):385-94.

15. Vijgen GH, Schouten R, Pelzers L, et al. Revision of laparoscopic adjustable gastric banding: success or failure? Obes Surg. 2012;22: 287-92.

16. Favretti F, Segato G, Ashton D, et al. Laparoscopic adjustable gastric banding in 1,791 consecutive obese patients: 12-year results. Obes Surg. 2007;17(2):168-75.

17. Stroh C, Hohmann U, Schramm H, et al. Fourteen-year long-term results after gastric banding. J Obes. 2011;2011:128451.

18. O'brien P, MacDonald L, Anderson M, et al. Long-term outcomes after bariatric surgery: fifteen-year follow-up of adjustable gastric banding and a systemic review of the bariatric surgical literature. Ann Surg. 2013;257(1):87-94.

19. Vinzens F, Kilchenmann A, Zumstein V, et al. Long-term outcome of laparoscopic adjustable gastric banding (LAGB): results of a Swiss single-center study of 405 patients with up to 18 years' follow-up. Surg Obes Relat Dis. 2017;13:1313-20. 
20. Miller K, Pump A, Hell E. Vertical banded gastroplasty versus adjustable gastric banding: prospective long-term follow-up study. Surg Obes Relat Dis. 2007;3:84-90.

21. Tolonen P, Victorzon M, Mäkelä J. 11-year experience with laparoscopic adjustable gastric banding for morbid obesity - what happened to the first 123 patients? Obes Surg. 2008;18:251-5.

22. Lanthaler M, Aigner F, Kinzl J, et al. Long-term results and complications following adjustable gastric banding. Obes Surg. 2010;20(8):1078-85.

23. Ramly EP, Safadi BY, Aridi HD, et al. Concomitant removal of gastric band and gastric bypass: analysis of outcomes and complications from the ACS-NSQIP database. Obes Surg. 2017;27:462-8.

24. Van Nieuwenhove Y, Ceelen W, Van Renterghem K, et al. Conversion from band to bypass in two steps reduces the risk for anastomotic strictures. Obes Surg. 2011;21:501-5.

25. Robert M, Poncet G, Boulez J, et al. Laparoscopic gastric bypass for failure of adjustable gastric banding: a review of 85 cases. Obes Surg. 2011;21:1513-9.

26. Ardestani A, Lautz D, Tavakkolizadeh A. Band revision versus Roux-en-Y gastric bypass conversion as salvage operation after laparoscopic adjustable gastric banding. Surg Obes Relat Dis. 2011;7(1):33-7.
27. Carr W, Jennings N, Boyle M, et al. A retrospective comparison of early results of conversion of failed gastric banding to sleeve gastrectomy or gastric bypass. Surg Obes Relat Dis. 2015;11(2):37984.

28. Chansaenroj P, Aung L, Lee WJ, et al. Revision procedures after failed adjustable gastric banding: comparison of efficacy and safety. Obes Surg. 2017;27(11):2861-7.

29. Carandina S, Maldonado P, Tabbara M, et al. Two-step conversion surgery after failed laparoscopic adjustable gastric banding. Comparison between laparoscopic Roux-en-Y gastric bypass and laparoscopic gastric sleeve. Surg Obes Relat Dis. 2014;10(6):108591.

30. Topart P, Becouarn G, Ritz P. Biliopancreatic diversion with duodenal switch or gastric bypass for failed gastric banding: retrospective study from two institutions with preliminary results. Surg Obes Relat Dis. 2007;3(5):521-5.

Publisher's Note Springer Nature remains neutral with regard to jurisdictional claims in published maps and institutional affiliations. 